\title{
Wave-vector-dependent Stoner approach to band ferromagnetism in Ni
}

\author{
N. C. Bacalis and N. Theodorakopoulos \\ Theoretical and Physical Chemistry Institute, National Hellenic Research Foundation, Vasileos Constantinou 48, \\ GR-116 35 Athens, Greece \\ D. A. Papaconstantopoulos \\ Complex Systems Theory Branch, Naval Research Laboratory, Washington, DC 20375-5345
}

(Received 14 November 1996)

\begin{abstract}
Recent experimental evidence [W. von der Linden et al., Phys. Rev. Lett. 71, 899 (1993)] that the exchange splitting in $\mathrm{Ni}$ follows the temperature dependence of the magnetization and provides strong support for the physical basis of the Stoner model of itinerant ferromagnetism. In this work, augmented plane wave results for paramagnetic and spin-polarized bands (supplemented by a single-parameter shrinking of the band splitting to fit the experimental value available at the symmetry point $L_{3}$ ) are used in a $k$-dependent, Stoner-like calculation, of the Curie temperature of $\mathrm{Ni}$. The calculated magnetization curve, saturation magnetization, Curie temperature, and specific heat coefficient are in agreement with experiment. [S0163-1829(97)12617-0]
\end{abstract}

The occurrence and strength of ferromagnetism in transition metals at zero temperature is well accounted for by first principles band structure calculations in the local density approximation (LDA); ${ }^{1}$ saturation magnetic moments for $\mathrm{Fe}$, $\mathrm{Co}$, and $\mathrm{Ni}$, differ by less than $10 \%$ from the corresponding experimental values (cf. Table I).

The finite-temperature behavior of metallic ferromagnets is usually described in terms of fluctuating band theories (FBT's), ${ }^{2}$ which attempt a synthesis between itinerant and localized models of magnetism; ${ }^{3-5}$ the degree of effective itineracy of magnetic moments can be described by the ratio of the saturation moment either to the (free) atomic moment, or to the paramagnetic moment determined by macroscopic measurents of the susceptibility above the Curie point. By either measure (cf. Table I), one expects the mechanism of magnetism in Ni to be itinerant dominated.

The first attempts ${ }^{2,3}$ to apply FBT's and related spinfluctuation descriptions to Ni produced significant deviations from experiment with respect to predicted values of $T_{c}$ [320 $\mathrm{K},{ }^{2} 1200 \mathrm{~K},{ }^{3} 450 \mathrm{~K}$ (Ref. 10) vs the observed value of 630 $\mathrm{K}]$, and/or shapes of the magnetization curve; ${ }^{3}$ a notable exception is the work of Mohn and Wohlfahrth, ${ }^{6}$ which presents an improved treatment of (paramagnetic) spin fluctuations that reduces the exchange integral and leads to a value of $T_{c}$ in agreement with experiment. Perhaps even more important, spin-fluctuation theories tend to produce serious underestimates of paramagnetic magnetic moments [e.g., $0.49 \mu_{B},{ }^{3} 0.42 \mu_{B}$ (Ref. 6)], which are not only much lower than the experimental value, but lie even below the saturation moment $\left[0.61 \mu_{B}\right.$ (Ref. 3)], i.e., in a regime not accessible even within a completely "localized" picture. 3,7

However, a recent paper by von der Linden et al. ${ }^{8}$ on spin-resolved photoemission presents strong experimental evidence in favor of the original Stoner ${ }^{9}$ model; these authors state that the influence of transverse fluctuations on the energy bands of $\mathrm{Ni}$ is negligible, and that the exchange splitting decreases with increasing temperature, closely following the magnetization. In the present work we demonstrate that in the case of $\mathrm{Ni}$, the most "itinerant" of ferromagnets (where local moments have been found to vanish ${ }^{10}$ ), obtaining a reasonable first-order estimate of $T_{c}$ should in principle be a matter of (a) correct zero-temperature energetics and (b) correct Stoner-like thermodynamics. Since the LDA, in spite of its success in estimating saturation magnetic moments, generally overestimates the values of exchange splittings, we try to address (a) in terms of a physically motivated empirical renormalization of LDA exchange splittings. Regarding (b), our work improves on previous approaches ${ }^{11,12}$ by using a Stoner-like interpolation scheme between paramagnetic and ferromagnetic band structures with $k$-dependent exchange splitting. Our simple one-electron, mean-field approach to the thermodynamics of band ferromagnetism, leads to a $\mathrm{Cu}$ rie temperature, a magnetization curve, a saturation moment,

TABLE I. Experimental and theoretical magnetic moments per atom for $\mathrm{Fe}, \mathrm{Co}$, and $\mathrm{Ni}$, in Bohr magnetons (Ref. 7); $p_{\text {sat }}$ is the saturated ferromagnetic value extrapolated to zero temperature; $\Delta n=p_{\text {sat }} / g$ is the experimental magneton number corrected by the spectroscopic splitting factor $g$, and thus corresponds more closely to the spin magnetization $M(0)$ (Ref. 17); $p_{\mathrm{LDA}}$ is the LDA value; $p_{\text {free }}=2 \sqrt{J(J+1)}$ is the free atom value calculated with $J=S ; p$ is a paramagnetic value determined in terms of the experimental slope, $p_{\text {para }}^{2} / 3$, of the Curie-Weiss susceptibility, and the relationship $p_{\text {para }}^{2}=p(p+g)($ Ref. 7$)$.

\begin{tabular}{|c|c|c|c|}
\hline & $\mathrm{Fe}$ & Co & $\mathrm{Ni}$ \\
\hline$p_{\text {sat }}$ & $2.22(19,20,18)$ & $1.72(20,18)$ & $0.62(19,18)$ \\
\hline$g$ & $2.10(17)$ & $2.18(17)$ & 2.21(17) \\
\hline$\Delta n$ & 2.11 & 1.58 & 0.56 \\
\hline$p_{\mathrm{LDA}}$ & $2.42(16)^{a}$ & $1.65(1)$ & $0.67(13)$ \\
\hline$p_{\text {free }}$ & 4.90 & 3.87 & 2.83 \\
\hline$p$ & $2.25(21)$ & 2.24(9) & $0.85(22)$ \\
\hline$p_{\text {sat }} / p$ & 0.99 & 0.77 & 0.73 \\
\hline$p_{\text {sat }} / p_{\text {free }}$ & 0.45 & 0.44 & 0.22 \\
\hline
\end{tabular}

${ }^{a}$ Calculated from Johnson's et al. original band structure, before the uniform increase of the exchange splitting of $56 \mathrm{mRy}$ (made by Johnson's et al. in order to match the experimental magnetization). 


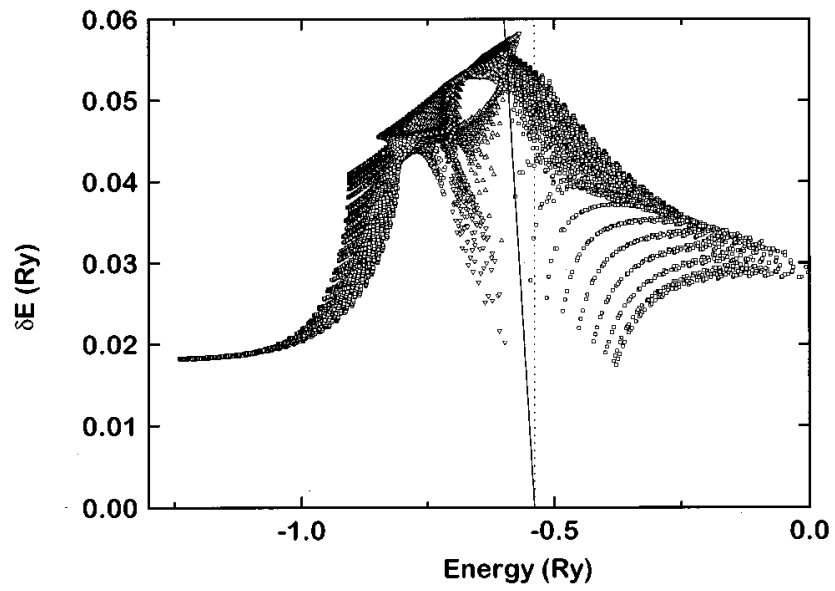

FIG. 1. The band splitting $\delta E=E_{k n}^{\downarrow}-E_{k n}^{\uparrow}$ is plotted against the energy $E_{k n}^{\uparrow}$ of the spin-up state for all values of $k, n$. States which fall within the triangle enclosed by the dotted line (drawn at the Fermi level), and the solid line [defined by $\delta E=-\left(E-E_{\text {Fermi }}\right)$ ] contribute to the net magnetization. The calculation (Refs. 1 and 13) includes six bands indicated (in order of increasing energy) by squares, circles, up and down triangles, diamonds, and squares.

and an electronic specific heat in agreement with experiment.

We use the results of zero-temperature APW band structure calculations ${ }^{1}$ as a starting point. At finite temperatures our interpolation between the ferromagnetic and the paramagnetic phase introduces effective bands by demanding thermal equilibrium of spin-up and spin-down electrons. The computational scheme makes no formal distinction between magnetically active and nonactive bands; in the spirit of an $a b$ initio, albeit approximate, calculation, we expect the difference to show up in the features of the band structure and/or the thermal occupation of the relevant states.

The spin-polarized band structure was calculated self-consistently ${ }^{13}$ via the von Barth-Hedin spin-polarized exchange and correlation potentials, ${ }^{14}$

$$
V_{\mathrm{xc}}^{ \pm}(\rho)=V_{\mathrm{KS}}(\rho)\left[B(\rho) \pm \frac{1}{3} A(\rho) \frac{\rho^{+}-\rho^{-}}{\rho}\right] \text {, }
$$

where $\rho^{+}, \rho^{-}$, and $\rho$ are the spin-down, spin-up, and the total electron charge densities, $V_{\mathrm{KS}}$ is the Kohn-Sham exchange potential, $B(\rho)=1+C x \ln (1+1 / x), \quad A(\rho)=1$ $+(C / 2) y \ln (1+1 / y), \quad x=r_{s} / 21, \quad y=x / 2^{4 / 3}, \quad r_{s}=(3 / 4 \pi \rho)^{1 / 3}$, and $C=0.045$. The spin-polarized band structure of $\mathrm{Ni}$ is shown in Fig. 2 of Ref. 13. We observe that the spin-up (majority) states are generally lower in energy than the spindown states and almost completely occupied, in contrast to the spin-down states. The exchange splitting near the Fermi level is apparent; it is already anticipated that high temperatures are needed in order to excite electrons from spin-up levels to unoccupied spin-down LDA levels. It should be noted however that LDA predictions for exchange splittings are often higher than experimental values, particularly in $\mathrm{Ni}$; for example, Eastman et al. ${ }^{15}$ have measured, by angle resolved photoemission, a splitting of $110 \mathrm{mRy}$ at $P_{4}$ in bcc Fe and $23 \mathrm{mRy}$ at $L_{3}$ in fcc $\mathrm{Ni}$, whereas the corresponding LDA values are $139 \mathrm{mRy}$ (Ref. 16) and $51 \mathrm{mRy},{ }^{13}$ respectively. Our work can account for this discrepancy by a

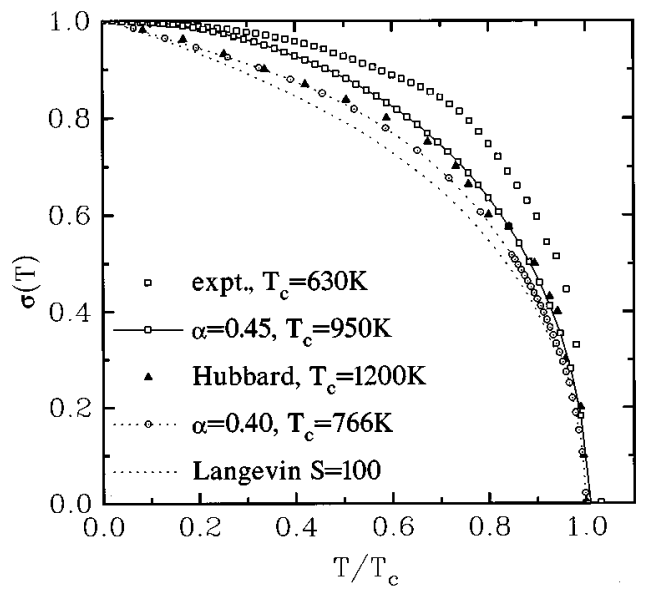

FIG. 2. The Ni magnetization curve: Experimental points (Ref. 18), results of our calculations for two values of the reduced exchange-splitting parameter $\alpha$, and Hubbard's theory (Ref. 3). $\alpha=0.45$ matches the experimental $L_{3}$ exchange splitting, as discussed in the text. $\alpha=0.40$ gives $T_{c}=766 \mathrm{~K}, M(0)=0.52 \mu_{B}$, $\gamma=5.8 \mathrm{~mJ} \mathrm{~mol}^{-1} \mathrm{~K}^{-2}$, and approaches the classical mean-field (Langevin) curve, also shown for comparison.

( $k$-dependent $)$ reduction of the exchange splitting, proportional to its LDA value at each $k$ point. This is achieved by a single parameter, $\alpha$, which matches the $L_{3}$ splitting to the experimental value, and results in a reasonable Curie temperature, $T_{c}$, for fcc $\mathrm{Ni}$, and a somewhat lower value for the saturation moment, also in agreement with experiment.

Moreover, the reduction of the exchange splitting leads to a correction of the specific heat coefficient $\gamma$ in the right direction: the values of $\gamma$ before and after the reduction, are 4.13 and 4.84, respectively, whereas the experimental value is $7.02 \mathrm{~mJ} \mathrm{~mol}^{-1} \mathrm{~K}^{-2} \cdot{ }^{17}$ In fact the theoretical value of $\gamma$ should be multiplied by the enhancement factor $1+\lambda$, previously calculated ${ }^{13}$ to be 1.24 due to electron-phonon interaction; this would raise the value of $\gamma$ to 6.0 , and provides further physical evidence for our simple parametric shrinking of exchange splitting.

The effective band scheme employed in this work is summarized as follows. For each $k$ point and for each band $n$, we define

$$
\epsilon_{k n}^{\uparrow, \downarrow}(T) \equiv \bar{\epsilon}_{k n}+\alpha \sigma(T)\left(E_{k n}^{\uparrow, \downarrow}-\bar{\epsilon}_{k n}\right),
$$

where $E_{k n}^{\uparrow, \downarrow}$, and $\bar{\epsilon}_{k n}$ are the LDA spin-polarized and paramagnetic band structures, respectively, calculated at the lattice constant of Ref. 13, $\sigma(T) \equiv M(T) / M(0)$ the reduced magnetization, and $\alpha$ is the exchange-splitting reduction parameter. Equation (2) incorporates both the experimentally observed temperature-dependent reduction of the exchange splitting $^{8}[$ via $\sigma(T)]$ and the zero-temperature experimental value of the band splitting at the symmetry point $L_{3}$ (Ref. 15) (via $\alpha$, which is the only adjustable parameter used in this work).

In Fig. 1 we have plotted the values of the LDA exchange splitting vs the energy of the spin-up state for all $k$ and $n$; it is evident that use of a single $k$-independent Stoner parameter, reflecting a $k$-independent exchange splitting, would be an oversimplification. 
The number of electrons in each subsystem is

$$
N^{\uparrow, \downarrow}(T)=\sum_{k n} \frac{1}{e^{\left[\epsilon_{k n}^{\uparrow, \downarrow}(T)-\mu(T)\right] / k T}+1}
$$

and the total number of electrons is

$$
N_{\text {total }}=N^{\uparrow}(T)+N^{\downarrow}(T) .
$$

At thermal equilibrium the chemical potential $\mu(T)$ is common and the magnetization is

$$
M(T)=N^{\uparrow}(T)-N^{\downarrow}(T) .
$$

Thus the simultaneous solution of Eqs. (4) and (5) for $\mu(T)$ and $M(T)$ provide the equilibrium value of the chemical potential and the magnetization at any desired temperature.

Figure 2 shows the reduced magnetization $\sigma(T)$ as computed in this work, the experimental values, ${ }^{18}$ and the curve calculated by Hubbard. ${ }^{3}$ We deduce from this calculation a Curie temperature $T_{c}=950 \mathrm{~K}, M(0)=0.53$ (for $\alpha=0.45$ ) and $T_{c}=766 \mathrm{~K}, M(0)=0.52$ (for $\alpha=0.40$ ). These values are in much better agreement with experiment $\left[T_{c}=630 \mathrm{~K}\right.$ and $M(0)=0.56]$ than those of either Ref. $3\left[T_{c}=1200 \mathrm{~K}\right.$ and $M(0)=0.6]$ or Ref. $11\left(T_{c}=2900 \mathrm{~K}\right)$. They also demonstrate the sensitivity of predicted Curie point on salient features of zero-temperature energetics (magnitude of exchange splittings) which hardly affect the saturation magnetization. This confirms our thesis that correct zerotemperature energetics play a decisive role in understanding itinerant ferromagnetism.

Results of similar calculations for $\mathrm{Fe}$ and $\mathrm{Co}$ (based on tight-binding spin-polarized band fitting ${ }^{1}$ rather than exact APW interpolation) lead to Curie temperatures of 5900 and $4100 \mathrm{~K}$ respectively, in disagreement with experiment and comparable to values found by Gunnarsson. ${ }^{11}$ This is not surprising since in $\mathrm{Fe}$ and $\mathrm{Co}$ ferromagnetism is dominated by local moments; indeed, it should be noted that the value of the ratio of $T_{c}^{\text {expt }} / T_{c}^{\text {theor }}$ is an increasing function of the degree of itineracy, as measured by the ratios in Table I.

Returning to Ni we wish to remark on the predicted critical behavior. Since our calculation is Stoner-like, it predicts mean-field behavior for the average magnetization as $T \rightarrow T_{c}$; surprisingly, however, it does more than that - as is evident from the good agreement of the magnetization curve with experiment near $T_{c}$. There is a crossover in the value of the magnetization critical exponent $\beta$ from $1 / 3$ to $1 / 2$ around $\left(T_{c}-T\right) / T_{c} \sim 0.05$, which closely parallels measurements of the internal field made in the critical regime, based on the Mössbauer effect ${ }^{23}$ and implying that some form of long-range interaction must be present. We have no way of ascertaining whether this detailed agreement is fortuitous or not; nonetheless, within the general physical picture of itinerant-dominated ferromagnetism (where the typical long-range dipolar corrections become ineffective), the experimentally observed asymptotic prevalence of mean-field behavior would necessitate some nondipolar form of residual long-range electronic correlation. Such correlations may in fact be implicitly included in our calculation, but this is something that only a complete theory of metallic ferromagnetism could decide. Our attempts serve only to demonstrate that a properly interpolated band-structure scheme combined with realistic zero-temperature energetics can give reasonable estimates of thermodynamic properties of itinerant ferromagnets (for $T \leqslant T_{c}$ ). In addition, the effective band scheme could be of direct use in a semiquantitative description of other temperature-dependent properties of $\mathrm{Ni}$, such as the recently observed Compton profiles ${ }^{24}$.

In conclusion we have proposed an empirical correction to the LDA value of the exchange splitting in $\mathrm{Ni}$ which using a Stoner model improves dramatically the calculated value of the Curie temperature. We emphasize that this approach is only appropriate for $\mathrm{Ni}$ due to its itinerant nature of magnetism. In $\mathrm{Fe}$ and $\mathrm{Co}$, which are characterized by local moment magnetism, our treatment is not valid and a spin-fluctuation theory is more appropriate. Our point of view is indeed consistent with the recent work of Uhl and Kübler ${ }^{25}$ who, using spin-fluctuation theory, obtain the Curie temperature $\left(T_{c}\right)$ of Fe to within $5 \%$ of experiment while they underestimate $T_{c}$ for $\mathrm{Ni}$ by $215 \mathrm{~K}$.

We are pleased to acknowledge discussions with L. L. Boyer, W. E. Pickett, D. J. Singh, and M. J. Mehl. One of us (N.C.B.) received partial support by NATO Grant No. CRG940118.
${ }^{1}$ D.A. Papaconstantopoulos, Handbook of the Band Structure of Elemental Solids (Plenum, New York, 1986).

${ }^{2}$ For example, C.S. Wang, R.E. Prange, and V. Korenman Phys. Rev. B 25, 5766 (1982), and Refs. 1-6 therein.

${ }^{3}$ J. Hubbard, Phys. Rev. B 23, 5974 (1981).

${ }^{4}$ T. Moriya, Spin Fluctuations in Itinerant Electron Magnetism (Springer, Berlin, 1985).

${ }^{5}$ J. B. Staunton, Rep. Prog. Phys. 57, 1289 (1994), and references therein.

${ }^{6}$ We recently became aware of the work of P. Mohn and E.P. Wohlfarth [J. Phys. F 17, 2421 (1987)], which is a significant exception.

${ }^{7}$ M.B. Stearns, in Numerical Data and Functional Relationships in Science and Technology, edited by H. P. J. Wijn, LandoltBörnstein New Series, Group III, Vol. 19a (Springer-Verlag, Berlin, 1986), p. 30.
${ }^{8}$ W. von der Linden, M. Donath, and V. Dose, Phys. Rev. Lett. 71, 899 (1993).

${ }^{9}$ E. C. Stoner, Proc. R. Soc. London, Ser. A 165, 372 (1938).

${ }^{10}$ J. B. Staunton and B. L. Gyorffy, Phys. Rev. Lett. 69, 371 (1992).

${ }^{11}$ O. Gunnarsson, J. Phys. F 6, 587 (1976).

${ }^{12}$ A. M. Oles and G. Stollhof, J. Magn. Magn. Mater. 54-57, 1045 (1986).

${ }^{13}$ J. R. Anderson, D. A. Papaconstantopoulos, L. L. Boyer, and J. E. Schirber, Phys. Rev. B 20, 3172 (1979).

${ }^{14}$ U. von Barth and L. Hedin, J. Phys. C 5, 1629 (1972).

${ }^{15}$ D. E. Eastman, F. J. Himpsel, and J. A. Knapp, Phys. Rev. Lett. 44, 95 (1980).

${ }^{16}$ W. B. Johnson, J. R. Anderson, and D. A. Papaconstantopoulos, Phys. Rev. B 29, 5337 (1984).

${ }^{17}$ C. Kittel, Introduction to Solid State Physics, 3rd ed. (Wiley, New York, 1966). 
${ }^{18}$ J. Crangle and G. M. Goodman, Proc. R. Soc. London, Ser. A 321, 477 (1971).

${ }^{19}$ H. Danan, A. Herr, and A. J. P. Meyer, J. Appl. Phys. 39, 669 (1968).

${ }^{20} \mathrm{R}$. Pauthenet, in Conference on High Field Magnetism, edited by M. Date (North-Holland, Amsterdam, 1983), p. 77.

${ }^{21}$ S. Arajs and D. S. Miller, J. Appl. Phys. 31, 986 (1960).
${ }^{22}$ S. Arajs and R. V. Colvin, J. Phys. Chem. Solids, 24, 1233 (1963).

${ }^{23}$ D. G. Howard, B. D. Dunlap, and J. G. Dash, Phys. Rev. Lett. 15, 628 (1965).

${ }^{24}$ D. I. Anastasopoulos and G. D. Priftis (unpublished).

${ }^{25}$ M. Uhl and J. Kübler, Phys. Rev. Lett. 77, 334 (1996). 